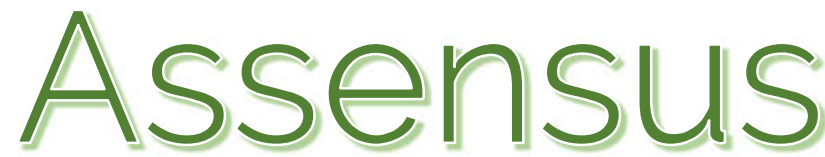

Revista de Investigación educativa y pedagógica

\title{
Estrategia "Educando mis emociones" para la prevención y disminución del bullying en estudiantes de Básica Secundaria
}

\section{Strategy "Educating my emotions" for the prevention and decrease of bullying in elementary school students}

\author{
Sandra milena Ojeda Madrid \\ Institución Educativa Marcealino Polo, \\ Colombia \\ sandraom1980@gmail.com
}

\author{
Liliana Rocío Agámez Díaz \\ Universidad de Córdoba, Colombia \\ lilianarocio90@hotmail.com
}

\section{Resumen}

Palabras claves:

Bullying, Agresor, Víctima, Emociones, Escuela.

\begin{abstract}
El presente estudio tuvo como propósito analizar la incidencia de la aplicación de una estrategia pedagógica social en la prevención y disminución del bullying en estudiantes de $8^{\circ}$ de básica secundaria de la Institución Educativa Marceliano Polo del municipio de Cereté - Córdoba. El estudio es de enfoque cualitativo y está enmarcado dentro del diseño de estudio de caso con una muestra de 36 estudiantes de octavo y 2 docentes. Los instrumentos fueron el formulario de encuesta, el formulario de entrevista y el diario de campo. Para el análisis de datos, se realizó una triangulación de la información, es decir, se articularon las entrevistas, encuestas y el desarrollo de la estrategia pedagógica social "Educando mis emociones" para llevar a cabo un análisis integral que permitió evidenciar los sentires de los estudiantes y percepciones de los docentes. Los resultados obtenidos pudieron establecer la caracterización de los estudiantes frente al bullying el cual se presenta en un alto porcentaje en las dimensiones Verbal y Social, en cuanto a la percepción de los docentes se demostró que conocen de la problemática de bullying pero no utilizan estrategias de prevención, por último la aplicación de la estrategia de inteligencia emocional contribuyó a mejorar el comportamiento y convivencia en los estudiantes, como también afrontar de mejor manera situaciones cotidianas y no cotidianas.
\end{abstract}

Recepción: Nov 29 de 2019 | Aceptación: Mar 04 de 2020 | Publicación: Dic 15 de 2020 
Revista de Investigación educativa y pedagógica

\begin{tabular}{ll}
\hline & Abstract \\
& The purpose of this study was to analyze the incidence of the \\
application of a social pedagogical strategy in the prevention and \\
reduction of bullying in 8th grade students of the Marceliano Polo \\
Educational Institution of the municipality of Cerete - Córdoba. The \\
study is of qualitative approach and is framed within the case study \\
design with a sample of 36 eighth grade students and 2 teachers. \\
The instruments were the survey form, the interview form and the \\
field diary. For the data analysis, a triangulation of the information \\
was done, in other words, the interview, the surveys and the \\
development of the social pedagogical strategy Ëducating my \\
Emotions" were assembled to carry out a comprehensive study \\
which showed students' thoughts and teachers' perceptions clearly. \\
The results obtained were able to establish the characterization of \\
the students against bullying which was in a high percentage in the \\
Bullying, \\
Aggressor,
\end{tabular}

Received: Nov 29, 2019 | Accepted: Mar 04, 2020 | Published: Dec 15, 2020 


\section{Introducción}

En el presente artículo se exponen los resultados de una investigación que buscó caracterizar las principales condiciones de riesgo asociadas a las manifestaciones de acoso escolar de los estudiantes de $8^{\circ}$ básica secundaria presentadas en el contexto, como también identificar las percepciones de docentes sobre las formas de relación e interacción de los estudiantes en los espacios de convivencia y estudio. Lo anterior, con el fin de aplicar una estrategia pedagógica social "Educando mis emociones" en la prevención y disminución del bullying. Todo esto dirigido a cumplir el objetivo general de analizar la incidencia de la aplicación de la estrategia pedagógica en la prevención y disminución del bullying en estudiantes de $8^{\circ}$ de básica secundaria de la Institución Educativa Marceliano Polo del municipio de Cereté - Córdoba.

La pertinencia del estudio se fundamenta en que en la actualidad la mayoría de las Instituciones Educativas públicas y privadas enfrentan situaciones relacionadas con problemas de convivencia escolar, matoneo y bullying; así también factores como el alto número de estudiantes por aula, las relaciones familiares conflictivas y el contexto educativo, afectan de forma directa las dinámicas al interior de las instituciones. En entrevistas con docentes, es común escuchar a algunos docentes expresar su preocupación por los problemas de convivencia escolar, principalmente por el desgaste y las dificultades para manejar los conflictos entre los educandos; por otro lado en revisión de los observadores y fichas de los estudiantes se evidencia como permanentemente en las oficinas de coordinación de convivencia y académica se vean colmadas de procesos disciplinarios con aquellos estudiantes que presentan comportamientos violentos y de bullying, que además de interrumpir los procesos de enseñanza/aprendizaje se pueden convertir en una amenaza para la convivencia escolar.

La Institución Educativa Marceliano Polo, contexto de la investigación, al igual que muchos otros planteles, también es un escenario en donde se evidencia bullying entre estudiantes. El colegio se encuentra ubicado en la parte céntrica del municipio de Cereté Córdoba, es de carácter oficial y tiene los cursos de preescolar hasta 11 de educación media. En cuanto al Proyecto Educativo Institucional se observa el modelo pedagógico tradicional, inclusivo, donde la evaluación es por competencia. La institución evalúa, a final de cada año, la contribución de los diferentes equipos en relación con el logro de los objetivos institucionales y con el fortalecimiento de un buen clima institucional. En el 2018, la autoevaluación institucional (ver análisis a profundidad en la tabla 1), en cuanto a clima escolar, se observaron resultados donde existen procesos en cada uno de sus componentes, pero en algunos casos no cumplen la verdadera función u objetivo que debiera, el mayor avance se encuentra solo en el papel o en evidencias fotográficas que podrían ser fácilmente manipulables para hacer ver una realidad que en verdad no existe. 
Todos estos aspectos, sustentan una problemática que afecta la convivencia escolar entre iguales, asunto que también se soporta con los apuntes encontrados en el observador de los estudiantes, y casos de seguimiento en la coordinación de convivencia y oficina de orientación escolar, donde se pueden percibir casos específicos de bullying. De igual forma, según lo expresado por los estudiantes, profesores y padres de familia, coinciden en la existencia del fenómeno con mayor prevalencia en los grados octavos de la institución educativa.

Tabla 1. Apreciación autoevaluación institucional 2018

\begin{tabular}{|c|c|c|c|}
\hline Componentes & Aspectos positivos & Aspectos por mejorar & Relación con el bullying \\
\hline Pertenencia y participación & $\begin{array}{lr}\text { Los } & \text { estudiantes } \\
\text { participan en juegos } \\
\text { inter-cursos y olimpiadas } \\
\text { externas }\end{array}$ & $\begin{array}{l}\text { No todos los estudiantes } \\
\text { participan en los distintos } \\
\text { equipos, no existen salidas } \\
\text { pedagógicas }\end{array}$ & $\begin{array}{l}\text { Es posible que se } \\
\text { presenten casos de } \\
\text { exclusión a estudiantes en } \\
\text { los distintos equipos }\end{array}$ \\
\hline Manual de convivencia & $\begin{array}{l}\text { Existe un manual de } \\
\text { convivencia }\end{array}$ & $\begin{array}{l}\text { El manual de convivencia no } \\
\text { está actualizado }\end{array}$ & $\begin{array}{l}\text { Al no estar actualizado no } \\
\text { hay normas o de existir no } \\
\text { son claras en el manejo de } \\
\text { acoso escolar o bullying }\end{array}$ \\
\hline Manejo de conflictos & $\begin{array}{l}\text { La institución programa } \\
\text { actividades orientadas a } \\
\text { reducir los conflictos }\end{array}$ & $\begin{array}{l}\text { No hay claridad acerca de } \\
\text { todas las competencias } \\
\text { requeridas para la convivencia, } \\
\text { en el año no se realizó } \\
\text { estrategias de convivencia } \\
\text { escolar }\end{array}$ & $\begin{array}{l}\text { No existen estrategias de } \\
\text { detección, manejo y } \\
\text { prevención de casos de } \\
\text { bullying. }\end{array}$ \\
\hline Índice de inclusión & $\begin{array}{l}\text { A partir del trabajo en } \\
\text { equipo se logra empezar } \\
\text { la construcción de un } \\
\text { plan de mejoramiento } \\
\text { que contempla en cada } \\
\text { uno de los componentes } \\
\text { de sus áreas de gestión } \\
\text { metas realizables sobre } \\
\text { educación inclusiva. }\end{array}$ & $\begin{array}{l}\text { Se encuentra en el papel, pero } \\
\text { su operatividad está en un } \\
20 \% \text {. Teniendo en cuenta que } \\
\text { no se llevan a cabo la cultura } \\
\text { inclusiva (red de apoyo, } \\
\text { talleres, diplomados) }\end{array}$ & $\begin{array}{l}\text { La inclusión hace parte del } \\
\text { mejoramiento de la } \\
\text { convivencia escolar, por lo } \\
\text { que se relaciona } \\
\text { directamente con el } \\
\text { tratamiento y la prevención } \\
\text { de bullying. }\end{array}$ \\
\hline
\end{tabular}

Nota. La apreciación que en la tabla se hace está basada en la autoevaluación institucional 2018 y observaciones realizadas por las investigadoras.

De acuerdo con la información de la tabla 1, y la revisio de otros documentos institucionales, son pocas las acciones tomadas por la Institución a fin de detectar, prevenir y tratar el bullying, el accionar docente frente a esto es poco operativo, limitándose a llamados de atención y remisión de casos al coordinador de convivencia con el fin de aplicar los correctivos según la normatividad existente, tales como la Ley 115 de 1994 y la Ley 1620 de 2013 donde se ha tratado de regular los procesos de convivencia a través de los manuales de convivencia escolar. En este punto, debe decirse que, lo que sucede muchas veces dentro y fuera de las aulas está alejado de lo que se espera con estas leyes.

Es preciso, resaltar la importancia del profesorado en la búsqueda de estrategias acordes que ayuden a superar todas estas dificultades de comportamiento en el aula o fuera de ella, es una labor donde también debe sentirse implicado toda la sociedad en general. Por tanto, 
es necesario que se incorporen estrategias pedagógicas orientadas hacia el desarrollo de la competencia social. De acuerdo con esto, se formuló esta pregunta de investigación: ¿Cómo incide la aplicación de una estrategia pedagógica social en la prevención y disminución del bullying en estudiantes de octavo grado de básica secundaria de la Institución Educativa Marceliano Polo del municipio de Cereté - Córdoba?. Para dar respuesta a la pregunta se desarrolló el estudio cuyos resultados se presentan en este artículo, siguiendo una metodología con enfoque cualitativo y un diseño de estudio de caso. Como resultados principales, se evidenció el cambio de comportamiento de los alumnos después de aplicada la estrategia social "Educando mis emociones" logrando prevenir los comportamientos de bullying y tratar los que ya existían. No solo durante la realización de la estrategia sino tiempo después de aplicada, también se desarrollaron las competencias ciudadanas y emocionales como la autoconciencia, autorregulación, empatía, habilidades sociales entre otras.

\section{Referentes teóricos}

En la actualidad se encuentran muchas investigaciones en cuanto al índice de prevalencia del bullying y sus efectos, pero específicamente en relación con estrategias sociales para su disminución y prevención en la básica secundaria en plano local se encuentran pocas investigaciones desarrolladas entre los años 2013 y 2018, aunque son más abundantes en el ámbito internacional.

Por ejemplo, se destaca la investigación de Garaigordobil et al., (2017) donde intervienen una problemática de acoso escolar en una Institución Educativa del Ecuador en el año 2015, cuyos resultados los comparan con un estudio en la misma institución, pero del año 2012 evidenciando diferencias significativas entre los dos valores, se observa una disminución del porcentaje de acoso escolar de un 10.6 \% que se presentó en el estudio inicial del (2012) a un 3.6\% en el año 2015. La estrategia de intervención se sustentó en la formación de los estudiantes en valores, tales como solidaridad, cooperación, amistad, paz y tolerancia y en conductas pro-sociales. En Latinoamérica, las investigaciones sobre aplicación de estrategias en contra del bullying son reducidos (Zych et al., 2015), los pocos estudios señalan que Colombia, con un 63\% (Román \& Murillo, 2011), es uno de los países en los que se registran mayores niveles de implicación en bullying, por encima del 51.1\% informado para 16 países de América Latina y del $29.2 \%$ reportado para 32 países europeos y EE. UU. (Chester et al., 2015; De Oliveira et al., 2015).

En Colombia, la mayoría de las investigaciones se han realizado en ciudades capitales como Bogotá, Cali, Medellín, Barranquilla, y, si bien aún es difícil establecer una prevalencia general debido a distintos factores, es posible reconocer que para la victimización la prevalencia oscila entre el 14.5 y el 69.2\%. En esta misma línea, pero más actualizado un estudio desarrollado por la Universidad de los Andes evidencia que en Colombia, cerca de 55 
mil estudiantes en 589 municipios del país en el marco de las Pruebas Saber se encontró que el $29 \%$ de los bachilleres de $5^{\circ}$ y el $15 \%$ de $9^{\circ}$ manifestaban haber sufrido algún tipo de agresión física o verbal por parte de algún compañero (Páez, 2013). Los datos anteriores, evidencian una problemática de convivencia social en la mayoría de Instituciones educativas, por lo que se requieren acciones, programas o estrategias que ayuden en la disminución o prevención de estos porcentajes.

\section{Algunos conceptos sobre el Bullying}

Expresiones como bullying, matoneo, matonismo y matonaje son términos de origen anglosajón, que en la actualidad se han empezado a utilizar con mayor frecuencia en nuestro contexto nacional y regional. La palabra "bullying" proviene del vocablo inglés que según el diccionario Oxford (2013) se encuentra "formado a partir del verbo to bully, intimidar". En este diccionario bully es definido como "a person who uses strength or influence to harm or intimidate those who are weaker", lo que equivaldría a definir a una persona que utiliza la fuerza o influencia de dañar o intimidar a los más débiles. Sin embargo, lo importante es entender el bullying como una agresión directa e indirecta hacia la víctima; "es indirecto cuando hay aislamiento social y exclusión deliberada de un grupo y directo, cuando hay ataques relativamente abiertos a la víctima" (Olweus, 1998 p. 26). De acuerdo con esto, queda claro que para hacer referencia al bullying tendrá que haber intimidación en un período de tiempo prolongado o de forma permanente, por lo que se podría concluir que otros tipos de manifestaciones agresivas o de violencia con las que se luchan en la escuela no son necesariamente expresiones de bullying, esto por no constituirse como una acción intencionada, repetitiva y sostenida, sino como situaciones de conflicto o desavenencias espontáneas y transitorias en el tiempo. En este sentido, la intimidación debe reunir al menos tres aspectos primordiales: el primero, es que sea un comportamiento agresivo o con la intención de realizar un daño al otro; el segundo, es que sea ejercido repetidamente y a lo largo del tiempo; y el último, es que se produzca un desbalance de poder entre los involucrados.

En este contexto, Goncy et al., (2015) mencionan que existen tres roles principales y son: acosadores, víctimas y espectadores, teniendo en cuenta que en numeras ocasiones se percibe a la intimidación como una relación uno a uno, es decir el estudiante que se cree el más fuerte, astuto o inteligente acosa a otro; pero en realidad existen otros actores como se menciona al inicio. Se puede afirmar, con certeza, que en este problema todos son víctimas ya que todos quedan con secuelas, unas leves y otras más graves, Previoces (2013) concuerda con esta afirmación al mencionar que los implicados en bullying "aprenden patrones de conducta que no los ayudará a desenvolverse en forma asertiva cuando sean adultos" (p. 22), de igual forma, hay consecuencias en la autoestima de cada uno de los implicados. Por último, es preciso señalar que el bullying es un fenómeno, una conducta que 
se aprende, es por ello que puede ser corregida por medio de estrategias pedagógicas, ya que señalar a los agresores no cambia las conductas, por el contrario, las realza.

\section{Sobre estrategias de prevención de bullying}

En cuanto a estrategias de prevención y tratamiento del bullying muchas investigaciones indican la necesidad de enfrentar el fenómeno del bullying por medio de procedimientos que impliquen la formación emocional (Hamodi \& Jiménez, 2018; Garaigordobil et al., 2017; Acosta \& Moreno, 2015; Goncy et al., 2015) entre otros. Las estrategias de prevención, pueden clasificarse en dos: prevención primaria y prevención secundaria (Hamodi \& Jiménez, 2018). Los primarios se proponen como estrategia de actuación antes de que ocurran las conductas violentas; promueve actitudes y conductas que favorecen la convivencia desde el momento en que se establecen las relaciones interpersonales. Por el contrario, los programas de prevención secundaria están dispuestos para controlar la violencia relacional ya existente. Se dirigen tanto a los agresores como a las víctimas y tratan de activar respuestas y comportamientos mejor adaptados con actividades sociales (Hamodi \& Jiménez, 2018). En la tabla 2, se describe el modelo escogido para el diseño de la estrategia de prevención y tratamiento del bullying en el marco de este estudio.

Tabla 2. Modelo y estrategia contra el Bullying

\begin{tabular}{|c|c|c|}
\hline Modelos & Estrategias & Descripción \\
\hline $\begin{array}{l}\text { Modelo de prevención centrado en el trabajo } \\
\qquad \text { con las emociones: } \\
\text { La forma en la que se sienten los } \\
\text { estudiantes, sus emociones y su propia } \\
\text { imagen es fundamental a la hora de } \\
\text { entender por qué actúan de una manera o de } \\
\text { otra. Esto pone de manifiesto que trabajando } \\
\text { todo el ámbito emocional desde los primeros } \\
\text { años podría resultar una forma beneficiosa } \\
\text { de crear una buena imagen personal y de } \\
\text { respeto hacia las demás personas. }\end{array}$ & Socioafectivo & $\begin{array}{l}\text { Si los estudiantes cuentan con una } \\
\text { inteligencia emocional sana, serán } \\
\text { capaces de desarrollar y controlar las } \\
\text { emociones para convivir de manera } \\
\text { respetuosa con los demás. Entre más } \\
\text { temprana la edad para trabajar la } \\
\text { inteligencia emocional será más efectiva, } \\
\text { ya que es cuando se empiezan a formar y } \\
\text { a trabajar emociones y sentimientos tan } \\
\text { importantes como la empatía }\end{array}$ \\
\hline
\end{tabular}

Fuente: Hamodi \& Jiménez (2018)

La estrategia pedagógica social se desprende del modelo de prevención centrado en el trabajo con las emociones, según los autores mencionados lo que se busca es potenciar lo afectivo y lo vivencial a la hora de abordar temas o problemas sociales. En relación Hamodi \& Jiménez (2018) menciona que este enfoque no desconoce lo cognitivo, sino que lo complementa con lo emocional y empático, permitiendo que, en el proceso educativo, la persona tome parte en su totalidad, con el objetivo de pasar por estas fases: sentir, pensar, actuar. Todo apunta también al desarrollo de competencias emocionales. 


\section{Competencias socioemocionales y conciencia social}

Autores como Colunga y García (2016) y Bisquerra et al., (2017), señalan que las competencias socioemocionales son el conjunto de conocimientos, capacidades y actitudes específicas que adquiere una persona a lo largo de toda su vida, para ser capaz de identificar, manejar, regular y expresar de forma satisfactoria sus emociones; al mismo tiempo que puede reconocer y comprender las circunstancias y las manifestaciones emocionales de las personas con las que se relaciona cotidianamente. De ahí que, en este estudio se parte del hecho que las competencias socioemocionales tienen relación directa en la personalidad de cada individuo, por lo tanto, la adquisición y el dominio de las competencias socioemocionales suponen bienestar y éxito en la esfera de lo personal y lo social, componente efectivo para el tratamiento y la prevención del bullying.

Po otra parte, la conciencia social se definen como el conjunto de habilidades de orden social que permiten a una persona relacionarse con otras de forma efectiva (Andrés, 2014). Aunque este constructo podría conceptualizarse desde distintos enfoques (humanitario, ambientalista, filantrópico) de los grupos sociales, en este estudio la conciencia social se asumió como la capacidad de identificar, comprender y atender -solidariamente-situaciones socio-afectivas donde estén implicadas otras personas. Cabe resaltar que esta parte de la competencia socioemocional se encuentra bastante ligada al concepto de Inteligencia Interpersonal propuesto por Gardner (1998). Así mismo, la conciencia social en este estudio, responde primordialmente a las siguientes capacidades: Habilidades sociales Básicas, autoconciencia y autoestima, autoconocimiento y automotivación, resolución de Conflictos, empatía, autocontrol y asertividad.

\section{Metodología}

Este estudio se enmarca dentro del enfoque cualitativo de paradigma hermenéutico donde el investigador ve al escenario y al objeto de estudio en una perspectiva holística. Los estados de conocimiento por su carácter valorativo e interpretativo del discurso giran alrededor del mencionado paradigma (Ramos, 2015), desde donde se propuso contribuir en la prevención y disminución del bullying en la básica secundaria de la Institución Educativa Marceliano Polo del municipio de Cereté - Córdoba. En concordancia, se siguió un diseño basado en estudio de casos, en donde se utiliza una metodología interpretativa que busca conocer y comprender en profundidad los casos que son objeto de análisis, los cuales se consideran complejos y que están en constante funcionamiento (Stake, 1999). Se hizo abordaje desde un estudio de caso único (Stake, 2005; Yin, 1989) ya que posee 
especificidades propias, que lo hace tener un valor en sí mismo y pretende alcanzar una mejor comprensión del caso concreto a estudiar.

\section{Técnicas de recolección de la información:}

Con relación a las técnicas, se utilizaron las siguientes:

La encuesta. Esta técnica de recolección de información se hizo necesaria para caracterizar las principales condiciones de riesgo asociadas a las manifestaciones de acoso escolar de los (36) estudiantes de $8^{\circ}$, para ello se construyó un instrumento (Cuestionario) en su estructura contiene veinte (20) preguntas cerradas, con tres opciones de respuesta (nunca - algunas veces - siempre) en 4 dimensiones de la categoría Bullying: Verbal, Físico, Psicológico y Social.

Entrevistas: se diseñó una entrevista semiestructurada con el propósito de identificar las percepciones de (2) docentes sobre las formas de relación e interacción de los estudiantes de $8^{\circ}$ de básica secundaria en los espacios de convivencia y estudio. El instrumento fue diseñado con 12 preguntas en la dimensión de Percepciones de la categoría Bullying.

Observación participante. Se utilizó un instrumento basado en notas de campo para registrar las observaciones realizadas por los investigadores durante y después de aplicada la estrategia "Educando mis emociones". Esto con el objetivo de analizar la incidencia de la aplicación de la estrategia pedagógica social en la prevención y disminución del bullying en estudiantes de $8^{\circ}$ de básica secundaria.

\section{Participantes}

Para el estudio fueron seleccionados 36 de los 113 estudiantes que conforman el grado octavo de educación básica secundaria y dos (2) de sus docentes de la Institución Educativa Marceliano Polo del Municipio de Cereté - Córdoba. Se tuvo en cuenta solo el grupo que presenta más dificultades en cuanto a convivencia escolar y antecedentes de bullying y que cumplían algunos criterios para la selección del caso (Taylor y Bogdan, 1987).

\section{Análisis y tratamiento de la información}

Para el análisis e interpretación de los datos, se definieron las categorías (ver tabla 3) que orientaron el procesamiento y análisis de la información recolectada que dio lugar a la construcción de sistemas categoriales permitiendo establecer las relaciones lógicas entre todas las categorías definidas. Aquí fue de gran importancia la triangulación, para garantizar la calidad de los datos y eliminando el sesgo de los investigadores. 
Tabla 3. Categorías y Dimensiones de la investigación

\begin{tabular}{|c|c|c|c|}
\hline $\begin{array}{l}\text { Objetivos } \\
\text { específicos }\end{array}$ & Categorías & Dimensiones & Definición \\
\hline $\begin{array}{l}\text { Caracterizar las } \\
\text { principales } \\
\text { condiciones de } \\
\text { riesgo } \\
\text { asociadas a las }\end{array}$ & \begin{tabular}{l}
\multicolumn{2}{c}{ Bullying } \\
Son conductas que \\
tienen que ver con \\
la intimidación, la \\
tiranización, el \\
aislamiento, la \\
amenaza, los
\end{tabular} & Verbal & $\begin{array}{l}\text { Previoces (2013) establecieron que los agresores utilizan } \\
\text { un vocabulario agresivo para expresar lo que siente, lo } \\
\text { que se quiere, lo que se piensa, a costa de los derechos } \\
\text { y los sentimientos de los demás, humillando y atacando } \\
\text { cuando no puede "salirse con la suya", fomentando la } \\
\text { culpa y el resentimiento de los otros. }\end{array}$ \\
\hline
\end{tabular}

manifestaciones insultos, de un

de acoso agresor sobre una

escolar de los víctima o víctimas

estudiantes de dentro o fuera de la

$8^{\circ}$ básica escuela, también es

secundaria la exclusión social

presentadas en como forma

el contexto agresiva de la

institucional relación social

(Goncy et al., 2015)

Físico

Previoces (2013) explicaron que: Los agresores dañan el cuerpo y la integridad psicológica de la víctima. Se divide en: (1) Violencia directa es la acción no accidental, que provoca daños físicos que puede ser catalogados por su nivel de gravedad como leves, moderadas o severas y (2) violencia Indirecta es aquella que consiste en ataques no direccionados al cuerpo de la víctima, sino a las pertenencias o a su libre tránsito por su lugar.

Previoces (2013) indicaron que es la violencia que merma, resquebraja, opaca emocionalmente atacando la autoestima mediante el desprecio, trato indigno y la falta de respeto hacia la otra persona, aumentando de esta forma el temor, el miedo, terror al agresor o el entorno donde se desarrolla las agresiones.

Social Es el maltrato que se le proporciona a la víctima delante de otras personas con el objetivo de aislar al individuo (Previoces, 2013)

Identificar las percepciones de docentes sobre las formas de relación e interacción de los estudiantes...
Según la psicología clásica de Neisser (2012) la percepción es un proceso activo-constructivo en el que el perceptor, antes de procesar la nueva información y con los datos archivados en su conciencia, construye un esquema informático anticipatorio, que le permite contrastar el estímulo y aceptarlo o rechazarlo según se adecue o no a lo propuesto por el esquema

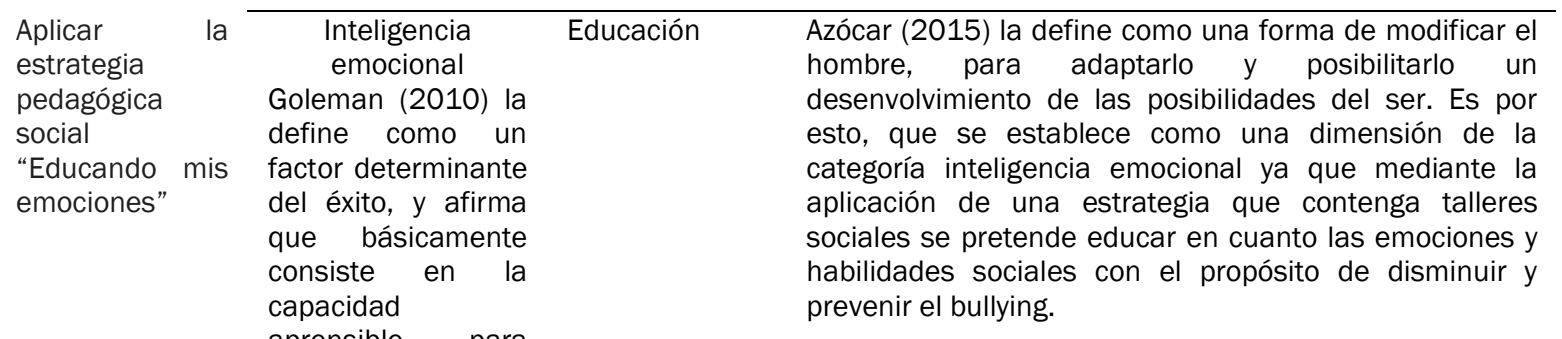

Fuente: Elaboración propia 
Así mismo, con cada uno de los instrumentos aplicados se llevó a cabo el siguiente procedimiento:

Encuesta. Después de recogido los datos con el instrumento se analizaron de la siguiente forma: 1- Organización de la frecuencia de respuestas de los (36) estudiantes a los (20) ítems en tablas con el programa Excel; 2- Se grafica las frecuencias en las tablas; 3- Se realiza el análisis inferencial y descriptivo según cada dimensión de la categoría Bullying: Verbal, Físico, Psicológico y Social.

Entrevistas: Al estar recolectadas las respuestas de los (2) docentes a las 12 preguntas del instrumento en una grabación, se siguió un análisis de contenido de la siguiente forma: 1Se redactó un resumen en físico de cada respuesta; 2- En este momento, se identificaron los temas centrales por párrafo, y se codificó; 3- Una vez codificados todos los documentos (unidades hermenéuticas), se formularon nuevas categorías; 4- Se organizan las categorías en mapas cognitivos; 5 - Se redactaron reflexiones según dimensión de Percepciones de la categoría Bullying.

Observación participante. Durante la aplicación de los talleres de la estrategia Educando mis emociones se registraron las observaciones en un instrumento de notas de campo, cuyo análisis se realizó de la siguiente forma: 1-Se sistematizaron las observaciones en un formato de ficha de sistematización diario de campo; 2- Se redactaron reflexiones haciendo triangulación con los antecedentes y teorías.

\section{Resultados}

A continuación, se presenta el análisis de los resultados obtenidos en cada una de las fases desarrolladas en la investigación. Al respecto, se hace una triangulación de la información, articulando las entrevistas, encuestas y el desarrollo de la estrategia pedagógica social "Educando mis emociones" atendiendo al tipo de investigación desarrollada para analizar la incidencia de la aplicación de la estrategia pedagógica en la prevención y disminución del bullying, Los resultados serán descritos a partir de los objetivos específicos propuestos en la investigación.

\section{Principales condiciones de riesgo asociadas a las manifestaciones de acoso escolar}

El primer objetivo se centró en caracterizar a través de una encuesta las principales condiciones de riesgo asociadas a las manifestaciones de acoso escolar de los estudiantes de $8^{\circ}$ básica secundaria presentadas en el contexto institucional: 
Tabla 4. Resultados síntesis de la encuesta

\begin{tabular}{lccc}
\multicolumn{1}{c}{ Dimensiones } & Nunca & Algunas veces & Siempre \\
\hline Dimensión: Verbal & 59 & 29 & 92 \\
Dimensión: Físico & 66 & 25 & 89 \\
Dimensión: Psicológico & 67 & 47 & 66 \\
Dimensión: Social & 61 & 27 & 92 \\
Total de la encuesta & 253 & 128 & 339
\end{tabular}

Fuente: Elaboración propia, producto del análisis de datos del estudio.

Figura 1. Gráfica resultados toda la encuesta.

\section{Síntesis de la encuesta}

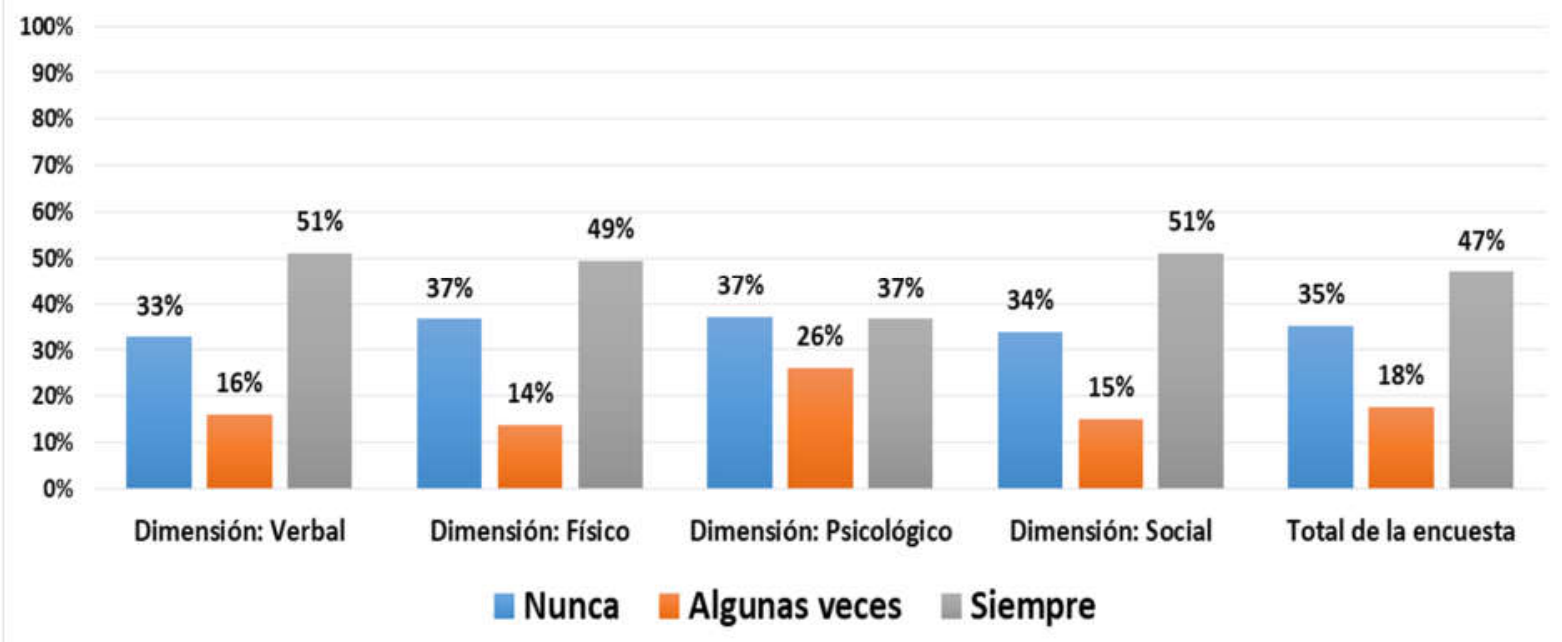

Fuente: Elaboración propia, producto del análisis de datos del estudio.

En la tabla 4 y figura 1 se aprecia el resultado total de la caracterización de las principales condiciones de riesgo asociadas a las manifestaciones de acoso escolar, es evidente entonces que hay serias manifestaciones de bullying por cuanto se observa que más del $40 \%$ de los estudiantes así lo perciben en cada una de sus dimensiones (Verbal, Física, Psicológica y Social) siendo el caso de la psicológica la de menos caracterización con un $37 \%$ y la verbal y social la de más caracterización con una incidencia del $41 \%$.

Todos estos datos de caracterización son coherentes con las investigaciones realizadas por (Acosta \& Moreno, 2015; Dewalt \& Dewalt, 2002; Hamodi \& Jiménez, 2018) cuando caracterizan los casos de bullying en escuelas y además coinciden en mencionar que el acoso escolar requiere atención por su asociación con uso y abuso de sustancias, abandono escolar, violencia intrafamiliar, embarazo adolescente, conducta delictiva, portación de armas, conducta suicida y trastornos psiquiátricos como déficit de atención, conducta oposicionista, ansiedad, problemas psicosomáticos y de conducta. 


\section{Percepciones de docentes sobre las formas de relación e interacción de los estudiantes}

Aquí se identificaron las percepciones de docentes sobre las formas de relación e interacción de los estudiantes de $8^{\circ}$ de básica secundaria en los espacios de convivencia y estudio. Para su alcance se diseñó y aplicó una entrevista semiestructurada con las siguientes preguntas:

Tabla 5. Preguntas de la entrevista

\begin{tabular}{|c|c|}
\hline Ítems & Preguntas \\
\hline 1 & ¿Para usted que es el Bullying? \\
\hline 2 & $\begin{array}{l}\text { ¿Ha percibido casos en los cuales estudiantes con características específicas son discriminados o maltratados } \\
\text { por sus compañeros? Explique }\end{array}$ \\
\hline 3 & $\begin{array}{l}\text { ¿Considera que el modelo de crianza en los hogares puede influir en la forma cómo se relacionan los } \\
\text { estudiantes en el colegio? Explique }\end{array}$ \\
\hline 4 & ¿Cómo percibe la forma de solucionar conflictos relacionados con violencia dentro de la institución? \\
\hline 5 & $\begin{array}{l}\text { ¿Piensa que la falta de normas claras y disciplina en el aula pueden generar actos violentos entre los } \\
\text { estudiantes? explique }\end{array}$ \\
\hline 6 & ¿El acompañamiento de los docentes durante el descanso favorece un ambiente libre de agresiones? explique \\
\hline 7 & ¿En qué grados observa más frecuencia actos de Bullying? \\
\hline 8 & ¿Inciden los medios de comunicación en el Bullying? \\
\hline 9 & ¿Ha trabajado la inteligencia emocional en los estudiantes? explique \\
\hline 10 & ¿En general cómo percibe la convivencia escolar en la institución? \\
\hline 11 & ¿Piensa que hay una relación entre educar las emociones y la disminución o prevención del Bullying? explique \\
\hline 12 & ¿En qué género se observa más frecuencia actos de Bullying? \\
\hline
\end{tabular}

Fuente: Elaboración propia.

En el mapa cognitivo de la figura 2, se aprecia de abajo hacia arriba encerrada en un primer recuadro rectangular la categoría en estudio (bullying), seguido la dimensión (Percepciones) la que apunta a dos docentes y desde donde sale cada indicador con recuadros azules y a la vez se conectan con categorías que emergieron de la entrevista, donde hay puntos de encuentro Existencia de bullying , Problemas de convivencia en el hogar, Falta de programas de prevención del bullying, No hay acompañamiento en los casos de acoso, En el grado 8-8 se evidencian más casos, En los hombres es más frecuente los casos de bullying.

Estos resultados indican que los docentes asocian la presencia de bullying a estado de ánimo, contextos familiares y contextos sociales de los estudiantes. De igual forma, los docentes apuntan a un grupo específico donde se presentan los problemas de convivencia escolar (8-8) y además su percepción sobre una estrategia pedagógica social relacionada con las emociones es positiva manifestando que se hace necesaria siendo esto coherente con los antecedentes consultados (Garaigordobil et al., 2017; Chester et al., 2015; De Oliveira et al., 2015) cuando afirman que la mejor estrategia para prevenir el bullying debe tener actividades sociales, que intervengan las emociones, el desarrollo de valores y la capacidad de convivir en armonía. 
De igual forma, se observa en las percepciones de los docentes que identifican al salón y al patio como los espacios escolares en los que se presenta con mayor frecuencia el acoso, coinciden estas percepciones con la investigación de (Hamodi \& Jiménez, 2018; Páez, 2013) donde se aprecia que el acoso se realiza en el aula y áreas del recreo cuando no hay control de parte de un adulto en este caso el docente.

Figura 2. Mapa cognitivo resultados percepción docente

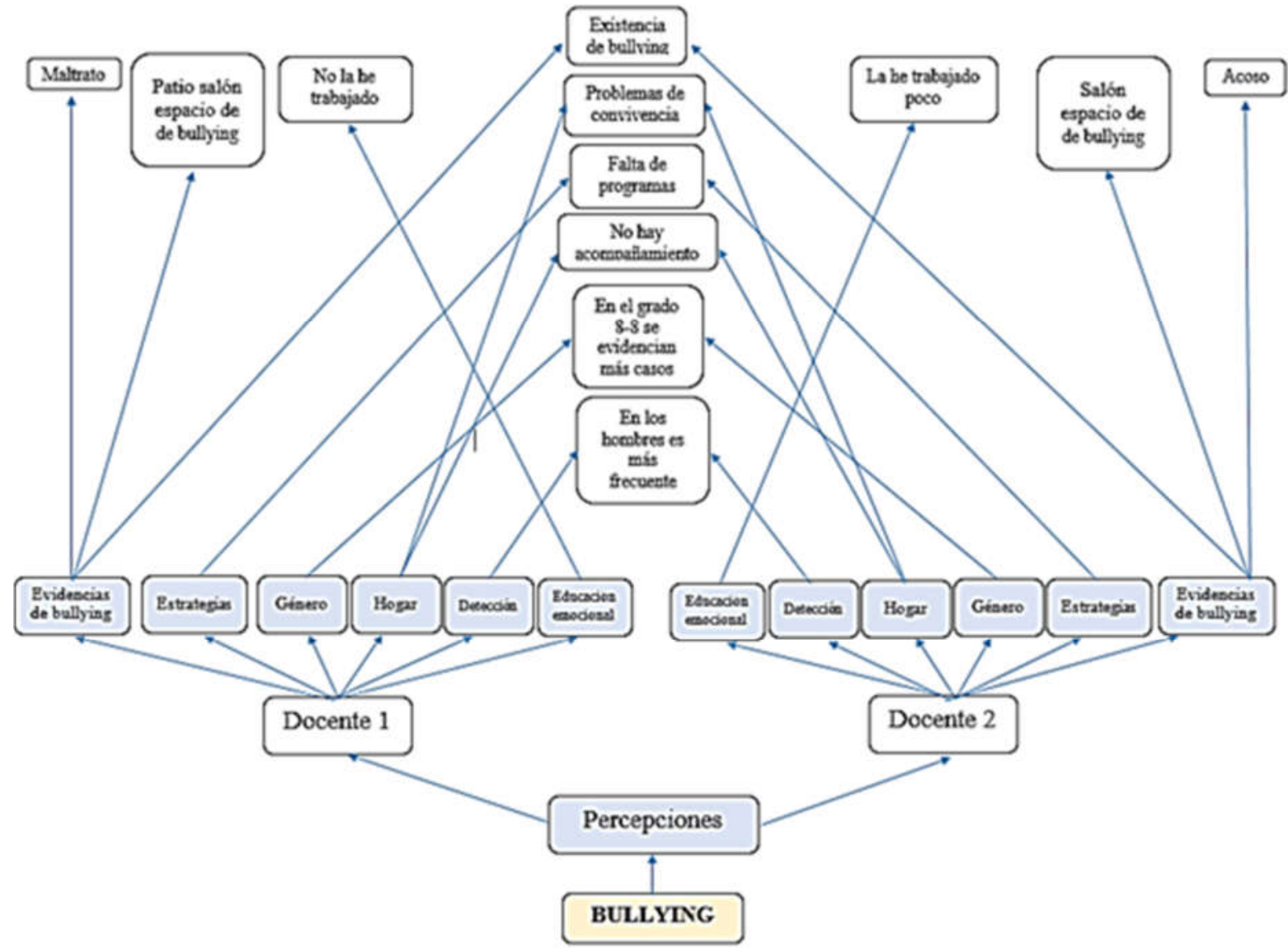

Fuente. Elaboración propia

Por último, los docentes mencionan diferentes aspectos relacionados con el bullying los cuales se ubican dentro del contexto social en donde se insertan las escuelas, por ejemplo, la ausencia de promoción de conductas sociales, en particular mencionaron la falta de programas para el desarrollo de valores y habilidades sociales

Desarrollo de la estrategia pedagógica social “Educando mis emociones" en la prevención y disminución del bullying

Se diseñó una estrategia social con el nombre "educando mis emociones" siguiendo el Programa de Educación Emocional para la Prevención de la Violencia, $2^{\circ}$ ciclo de ESO, de 
autoría Caruana (2005) y utilizó como herramienta una serie de talleres los que se describen a continuación:

Tabla 5. Estrategia pedagógica social

\begin{tabular}{|c|c|c|c|}
\hline \multicolumn{4}{|c|}{ Estrategia pedagógica social } \\
\hline \multirow{2}{*}{\multicolumn{4}{|c|}{$\begin{array}{l}\text { Nombre: Educando mis emociones } \\
\text { Propósito: Crear conciencia para la prevención y disminución del bullying, al igual que el fortalecimiento de la } \\
\text { inteligencia emocional de los estudiantes a través de } 7 \text { talleres de educación emocional. }\end{array}$}} \\
\hline & & & \\
\hline \multicolumn{4}{|c|}{ Descripción de las actividades (Talleres) } \\
\hline $\mathrm{N}$ & Nombre & Competencia Socioemocional & Duración \\
\hline 1 & La balanza & Autoconciencia y Autoestima & 2 horas \\
\hline 2 & Pantallas de proyección & Autoconciencia y Empatía & 2 horas \\
\hline 3 & Paseo por el bosque & Autoconocimiento y Automotivación & 2 horas \\
\hline 4 & Barómetro de valores & Autoconciencia y Habilidades Sociales & 2 horas \\
\hline 5 & Te aprecio - me aprecias & Autocontrol y Autoconciencia & 2 horas \\
\hline 6 & Expresando críticas de forma adecuada & Autocontrol y Asertividad & 2 horas \\
\hline$\overline{7}$ & Lectura del pensamiento & Empatía y Habilidades Sociales & 2 horas \\
\hline \multicolumn{4}{|c|}{ Momentos } \\
\hline 1 & \multicolumn{3}{|c|}{ Socialización de la estrategia con los estudiantes } \\
\hline 2 & \multicolumn{3}{|l|}{ Aplicación de talleres } \\
\hline 3 & \multicolumn{3}{|l|}{ Aplicación actividades del taller } \\
\hline 4 & \multicolumn{3}{|c|}{ Registro de comportamientos durante el taller } \\
\hline 5 & \multicolumn{3}{|c|}{ Registro de comportamientos después de aplicado el taller } \\
\hline
\end{tabular}

Fuente: Elaboración propia.

Los resultados sobre la aplicación de la estrategia pedagógica social "Educando mis emociones" en la prevención y disminución del bullying en estudiantes de $8^{\circ}$ de básica secundaria de la Institución Educativa Marceliano Polo del municipio de Cereté por medio de 7 talleres que fortalecen las competencias emocionales fueron significativos, se evidencia que en el primer taller titulado La balanza los estudiantes se mostraron apáticos y poco motivados, durante su desarrollo, hacían desorden, bulla, e incluso algunos comportamientos que bien podrían llamarse bullying, pero gracias al docente acompañante se pudo controlar a tiempo todos estos problemas de convivencia escolar.

Haciendo la triangulación con la encuesta de caracterización, las entrevistas de percepción y la teoría, se observa que no es extraño el comportamiento inicial, pues se aprecia en este caso de estudio que el resultado total de la caracterización de las principales condiciones de riesgo asociadas a las manifestaciones de acoso escolar, es evidente que hay seria incidencia del bullying; por cuanto se observa que más del $40 \%$ de los estudiantes lo perciben en cada una de sus dimensiones (Verbal, Física, Psicológica y Social), también en la 
entrevista a los docentes se observa que coinciden en sus respuestas frente a la sub-categoría Bullying en la Escuela, su percepción es que en el grado 8-8 es donde más se presenta el caso en estudio. Al respecto Bisquerra y Hernández (2017), manifiestan que la educación emocional en un inicio no es significativa, es un tanto difícil de que los estudiantes se acostumbren a un ritmo de trabajo diferente, por cuanto este es un proceso educativo, continuo y permanente, que pretende potenciar el desarrollo de las competencias emocionales como elemento esencial para prevenir y tratar el bullying. Posterior al primer taller, aún persistían comportamientos enmarcados en el bullying.

Posteriormente, en el segundo taller denominado Pantallas de proyección, ya se nota un cambio en el comportamiento de los estudiantes, están más ansiosos y atentos. Después de unos días de aplicado el taller se informa de un cambio en los estudiantes; a través de la caracterización y encuesta se pudo determinar la presencia de indisciplina y casos graves de bullying, aunque los provocadores o victimarios eran pocos (de 3 a 4) estudiantes. En relación con esto Previoces (2013), menciona que los agresores escolares presentan desórdenes. Estos desórdenes, sin llegar a ser trastornos mentales graves, dificultan sus relaciones sociales y su modo de dilucidar la realidad es tardío.

De este modo, el cambio en el comportamiento en los estudiantes se fue dando con la aplicación de los talleres siguientes $(3,4,5,6$ y 7) donde era evidente la transformación en las relaciones. Los resultados concuerdan con el estudio de Torrego (2011) el cual aplicó un módulo integrado para la gestión de los conflictos de convivencia desde una perspectiva de centro, lo cual desde esta investigación aporta un nuevo elemento, ya que muestra al estudiante como eje mediador de los conflictos.

Por último, al analizar el caso en estudio mediante la categoría 2 (Inteligencia emocional) se aprecia cómo esta influye positivamente en el comportamiento, logrando prevenir los comportamientos de bullying y tratar los que ya existían. No solo durante la realización de los talleres también después de realizados, teniendo en cuenta que se pasó por los salones en fechas posteriores al desarrollo de la estrategia, logrando apreciar evidencias que demuestran una excelente convivencia, además los estudiantes mejoraron en su rendimiento académico aunque no era el objetivo de la investigación, así como también se desarrollaron las competencias ciudadanas y emocionales como la autoconciencia, autorregulación, empatía, habilidades sociales, entre otras.

\section{Conclusión}

En primer lugar se caracterizaron las principales condiciones de riesgo asociadas a las manifestaciones de acoso escolar de los estudiantes de $8^{\circ}$ básica secundaria presentadas en el contexto institucional, donde es evidente que existían serias manifestaciones de bullying 
dado que más del $40 \%$ de los estudiantes así lo perciben en cada una de sus dimensiones (Verbal, Física, Psicológica y Social), siendo el caso de la psicológica la de menos caracterización con un 37\% y la verbal y social la de más caracterización con una incidencia del 41\%. Todos estos datos de caracterización son coherentes con las investigaciones realizadas por Hamodi y Jiménez (2018), Acosta y Moreno (2015), Dewalt y Dewalt (2002), cuando caracterizan los casos de bullying en escuelas y además coinciden en mencionar que el acoso escolar requiere atención por su asociación con uso y abuso de sustancias, abandono escolar, violencia intrafamiliar, embarazo adolescente, conducta delictiva, portación de armas, conducta suicida y trastornos psiquiátricos como déficit de atención, conducta oposicionista, ansiedad, problemas psicosomáticos y de conducta.

En segundo lugar, se concluye que los datos obtenidos permitieron una representación de las percepciones sobre el bullying. Indicando que los docentes asocian la presencia de este al estado de ánimo, contextos familiares y contextos sociales de los estudiantes. De acuerdo a lo manifestado, los estudiantes víctimas de acoso presentan baja autoestima, al igual que los acosadores, además estos últimos, presentan en gran parte problemas en el núcleo familiar; maltrato físico o verbal, desinterés por parte de los padres, deficiencias económicas. También, tienen la concepción de que el bullying se caracteriza por la intencionalidad de agredir de cualquier forma a alguien, que es víctima, mientras los victimarios presentan una preferencia a iniciar, y perpetuar, situaciones donde las víctimas están en una situación indefensa. Su percepción sobre una estrategia pedagógica social relacionada con las emociones es positiva manifestando que se hace necesaria. En este sentido, se observa que identifican al salón y al patio como los espacios escolares en los que se presenta con mayor frecuencia el acoso, coinciden estas percepciones con la investigación de (Hamodi \& Jiménez, 2018) donde se aprecia que el acoso se realiza en el aula y áreas del recreo cuando no hay control de parte de un adulto, en este caso el docente.

En tercer lugar, se logró el último objetivo específico propuesto, al aplicar la estrategia pedagógica social "Educando mis emociones" mediante 7 talleres de inteligencia emocional, se evidencia que los estudiantes mejoraron en su comportamiento, relaciones interpersonales, convivencia, inclusión, entre otros aspectos de las dimensiones Verbal, Física, Psicológica y Social. Al ser una estrategia social, durante los momentos de la aplicación de los talleres el docente observa motivados y orientados a los grupos, el trabajo es colaborativo, pero también de trabajo autónomo, al principio algunos estudiantes se sentían desmotivados y algunos apáticos, pero luego se adaptaron fácilmente. De forma transversal los estudiantes fueron más disciplinados y responsables en las actividades académicas de las distintas asignaturas.

El estudio desarrollado difiere de los antecedentes expuestos en el manejo metodológico de investigación, ya que adopta una estrategia con talleres desde el desarrollo de la inteligencia emocional y el diseño metodológico de estudio de casos. Los resultados demuestran el impacto positivo de la investigación ya que refleja una solución viable a las 
necesidades socio-educativas ya sea local, regional o nacional teniendo en cuenta que la convivencia escolar y el manejo de conflictos hace un ambiente agradable de aprendizaje (Calle et al., 2011). El uso de estrategias mediadoras de las emociones requirió en los estudiantes construcciones cooperativas y de inclusión, como lo menciona (Torrego, 2011; Román \& Murillo, 2011) estas características además de la prevención y tratamiento del bullying también desarrollan competencias emocionales, ciudadanas, sociales de forma inherente a los objetivos propuestos.

\section{Referencias}

Acosta, R., \& Moreno, L. (2015). Estrategias de convivencia escolar para la prevención del bullying en la Escuela Técnica “Los Magallanes" en el estado Carabobo (Bachelor's thesis).

Andrés, M. (2014). Conductas de ciberacoso en niños y adolescentes. Hay una salida con la educación y la conciencia social. Educar, 50(2), 383-400.

Azócar, R. (2015). La visión epistemológica de la educación. Documento online. https://www.aporrea.org/educacion/a207491.html

Bisquerra, R., \& Hernández, S. (2017). Psicología positiva, educación emocional y el programa aulas felices. Papeles del Psicólogo, 2017, vol. 38, num. 1, p. 58-65. https://doi.org/10.23923/pap.psicol2017.2822

Calle, M., De Cleves, N., \& Burgos, M. (2011). Incidencia de la inteligencia emocional en el proceso de aprendizaje. Nova, 9(15), 94-106.

Caruana, A. (2005). Programa de educación emocional y prevención de la violencia - $2^{\circ}$ ciclo de la ESO. Generalitat Valenciana, Conselleria de Cultura, Educació i Esport.

Chester, M. Callaghan, A. Cosma, P. Donnelly, W. Craig, S. Walsh, M. Molcho. (2015). Cross-national time trends in bullying victimization in 33 countries among children aged 11, 13 and 15 from 2002 to 2010European Journal of Public Health, 25 (2) , pp. 61-64

Colunga Santos, S., \& García Ruiz, J. (2016). Intervención educativa para desarrollar competencias socioemocionales en la formación académica. Humanidades Médicas, 16(2), 317-335.

De Oliveira, M.A. Silva, F.C. Mello, D.L. Porto, A.C. Yoshinaga, D.C. Malta. (2015). The causes of bullying: Results from the National Survey of School Health (PeNSE). Revista Latino-Americana de Enfermagem, 23 (2), pp. 275-282

DeWalt, Kathleen M. \& DeWalt, Billie R. (2002). Observación participante: Guía para trabajadores de campo. Walnut Creek, CA: AltaMira Press.

Garaigordobil, M., Martínez-Valderrey, V., \& Machimbarrena, J. M. (2017). Intervención en el bullying y cyberbullying: Evaluación del caso Martín. Revista de Psicología Clínica con Niños y Adolescentes, 4(1), 25-32. 
Gardner, H. (1998). Inteligencias múltiples. Paidós.

Goleman, D. (2010). Inteligencia social: la nueva ciencia de las relaciones humanas. Editorial Kairós.

Goncy, E. A., Sutherland, K. S., Farrell, A. D., Sullivan, T. N., \& Doyle, S. T. (2015). Measuring teacher implementation in delivery of a bullying prevention program: The impact of instructional and procedural adherence and competence on student responsiveness. Prevention science, 16(3), 440-450.

Hamodi Galán, C., \& Jiménez Robles, L. (2018). Modelos de prevención del bullying:¿ qué se puede hacer en educación infantil?. IE Revista de investigación educativa de la REDIECH, 9(16), 29-50.

Neisser, U. (2012). Percepción. Recuperado de: http://www. um. es/pguardio/documentos/percepción. pdf.

Olweus, D. (1998). Conductas de acoso y amenaza entre escolares. Morata.

Dictionary, O. (2013). Oxford dictionary. nd Performance. Accessed.

Páez, A. (2013). Proyecto de Ley Orgánica de Prevención, Control y Sanción del Acoso, Intimidación o Violencia en los centros de estudio del Ecuador (Bullying). 20, 17-19.

Previoces (2013). (Programa de intervención de la violencia en la convivencia escolar). Guía docente para prevenir la violencia escolar.

Ramos, C. (2017). Los paradigmas de la investigación científica. Avances en psicología, 23(1), 9-17. https://doi.org/10.33539/avpsicol.2015.v23n1.167

Román, M. \& Murillo, J. (2011). Latin America: School bullying and academic achievement. Cepal Review, 104, pp. 37-53

Stake, R. (1999). Investigación con estudio de casos. Morata.

Stake, R. (2005). Investigación con estudio de casos. (3ra Ed.). Morata

Yin, R. (1989). Case Study Research: Design and Methods. Sage.

Zych, I. \& Ortega-Ruiz, R. (2015). Scientific research on bullying and cyberbullying: Where have we been and where are we going. https://doi.org/10.1016/j.avb.2015.05.015

Mora, A. (2014). Propuesta para la elaboración de un manual para la gestión de los conflictos estudiantiles. Gestión de la educación, 1-35. 